\title{
Ecologia de mosquitos (Diptera, Culicidae) em criadouros naturais e artificiais de área rural do norte do Paraná, Brasil. VIII. Influência das larvas predadoras (Toxorhynchites sp., Limatus durhamii e Culex bigoti) sobre a população de larvas de Culex Quinquefasciatus e Culex eduardoi
}

José Lopes ${ }^{1}$

\begin{abstract}
Ecology of mosquitoes (Diptera, Culicidae) in natural and artificial rural breeding sites in northern Paraná State, Brazil. VIII. The influence of predator larvae (Toxorhynchites sp., Limatus durhamii and Culex bigoti) on the populations of Culex quinquefasciatus and Culex eduardoi. Larvae of Culex eduardoi, Culex bigoti, Limatus durhamii and Toxorhynchites were collected in a tyre that had been placed in a wood by a river bank. These larvae were present coexisting and in an individualized form. It was observed that Toxorhynchites, Culex bigoti and Limatus durhamii were efficient in reducing the other two species's population. Culex bigoti and Toxorhynchites showed preference for Culex quinquefasciatus larvae, while Limatus durhamii preferred Culex eduardoi. The most efficient predator was Toxorhynchites, and the least efficient was Limatus durhamii. The reduction of the preys' population density was statistically meaningful in the face of the three species considered as predators.

KEY WORDS. Culicidae, biological control, predators, larvae, artificial breeding sites
\end{abstract}

Culex bigoti Bellardi, 1862 e as espécies de Toxorhynchites Theobaldi apresentam em suas fases larvárias o comportamento alimentar predatório. Sabetíneos, embora possam predar, são considerados predadores não obrigatórios, isto é, usam esse método de alimentação quando falta alimento no criadouro ou em encontros casuais com a presa (MACHADO-ALLISON 1981).

Machos e fêmeas adultos de Toxorhynchites alimentam-se de néctar das flores (HuTCHINGS 1995), enquanto as fềmeas de Culicini e Sabethini são hematófagas. Assim, somente Toxorhynchitini são considerados para o estudo de controle biológico.

Muitos trabalhos foram executados em laboratório visando à determinação da viabilidade do uso de Toxorhynchites como agente de controle biológico (O'Flynn \& Graig 1982; CAstner \& Bailey 1984; HubBard et al. 1988). A eficiência desse agente de controle biológico em criadouros de pequeno porte está evidenciada por várias pesquisas (Focks et al. 1982; RUSSO 1983, 1986; BAILEY et al. 1983). Desta forma, tentativas foram realizadas visando à criação massal em laboratório e liberação em campo para atuarem como agentes capazes de diminuir a densidade populacional de larvas pertencentes a espécies vetoras de doenças (BAILEY et al. 1983; TOOHEY et al. 1985; ANNIS \& RUSMIARTO 1988).

1) Departamento de Biologia Animal e Vegetal, Universidade Estadual de Londrina. Caixa Postal 6001, Campus Universitário, 86051-970 Londrina, Paraná, Brasil.

E-mail: jea@uel.br 
A presente pesquisa teve como objetivo verificar a eficiência de Toxorhynchites, Culex bogoti e Limatus durhamii Thobaldi, 1901 em diminuir a população de larvas de Culicidae observadas em pneus alocados em mata ciliar.

\section{MATERIAL E MÉTODOS}

Foram instalados pneus de automóvel em cinco pontos de coleta, no interior da mata ciliar do Ribeirão São Domingos, distantes $2 \mathrm{~km}$ um do outro. Este ribeirão tem sua nascente no Município de Cambé e foz, no Ribeirão Cafezal, Município de Londrina, Estado do Paraná, sul do Brasil. A mata ciliar está reduzida e caracteriza-se por estado de regeneração.

Em cada ponto de coleta, foi instalado um pneu na posição vertical, amarrado ao tronco de uma árvore, abastecido com dois litros de água de poço. As coletas foram quinzenais por um período de 12 meses, sendo retirado todo o conteúdo com auxílio de uma bomba de sucção e concomitantemente coado em uma peneirinha com malha de $200 \mu \mathrm{m}$ de diâmetro. Todas as larvas e pupas foram introduzidas em frasco de transporte contendo água de poço e a água coada foi recolocada no criadouro.

No laboratório, as larvas foram quantificadas e colocadas para criar. A identificação foi feita baseada nas exúvias das larvas de quarto estágio e nos seus respectivos adultos.

A quantificação das larvas das diferentes espécies e a comparação do tamanho de suas populações, dos recipientes com e sem a presença de larvas predadoras, foi o principal parâmetro usado para inferir sobre a eficiência de predação de cada espécie.

Como o objetivo foi estudar o comportamento natural das populações de presa e predador, sem inferir no criadouro com padronizações, coletou-se e computou-se as larvas independentes dos ínstares em que se encontravam, incluindo inclusive as pupas.

Para comprovação estatística o Teste de Análise de variância foi usado para detectar diferenças entre o tamanho da população da presa nos momentos onde está estava coabitando com os predadores e na ausência destes. O Teste de Tukey foi usado para estabelecer a condição que se diferenciava das demais. Para a análise final, os dados quinzenais foram transformados em Média de Willians, devido a presença de muitos zeros.

Para comprovar a ação de Limatus durhamii sobre a população de Culex quinquefasciatus Say, 1823 e Culex eduardoi Casal \& Garcia, 1968, considerou-se estas duas espécies como presa, e o número de larvas, em cada coleta, foram somadas, originando uma única observação e depois transformadas em Média de Willians

Melhores informações sobre a área de estudo, descrição do recipiente e metodologia de coleta, poderão ser obtidas em LOPES et al. (1995).

\section{RESULTADO E DISCUSSÃO}

Além de Toxorhynchites sp., Culex bigoti, em estágio imaturo, apresenta hábito alimentar carnívoro (ForATTINI 1965; LOUNIBOS et al. 1987). Junta-se estas duas, as de Limatus durhamii cujas larvas, embora não sejam obrigatoriamente 
predadoras como alguns outros sabetíneos, comportam-se como tais em caráter não obrigatório e principalmente na ausência de alimento no criadouro (LOPES et al. 1985a).

Observa-se na tabela I o número médio de larvas de Culex eduardoi, Culex quinquefasciatus e Limatus durhamii, quando estas foram encontradas sós ou coabitando com espécies predadoras. O número médio de larvas de Culex eduardoi e Culex quinquefasciatus coletadas em pneu é significativamente maior quando coletada na ausência do predador. O número de repetições foi suficiente para suspeitar que houve a ação do predador, influenciando na diminuição da população das duas espécies consideradas presas, em detrimento das possíveis influências ambientais ou atividade de postura de ovos.

Tabela I. Número de repetições $(N)$, médias aritmética $(X)$ e de Willians $(X w)$ da quantidade de larvas das espécies consideradas presas (Culex quinquefasciatus, Culex eduardoi), isoladas e quando estavam coexistindo com as larvas predadoras (Limatus durhamii, Culex bigoti, Toxorhynchytes), em criadouros artificiais na mata ciliar de um ribeirão no norte do Paraná, Brasil.

\begin{tabular}{lrrc}
\hline \multicolumn{1}{c}{ Espécies } & $\mathrm{N}$ & $\mathrm{X}$ & $\mathrm{XW}$ \\
\hline C. eduardoi & 34 & 97,60 & 1,64 \\
C. eduardoi + L. durhamii & 41 & 14,40 & 0,43 \\
C. eduardoi + C. bigoti & 13 & 47,50 & 1,15 \\
C. eduardoi + L. durhamii + C. bigoti & 7 & 13,40 & 0,72 \\
C. eduardoi + L. durhamii + Toxorhynchites sp. & 7 & 9,30 & 0,48 \\
C. quinqufasciatus & 18 & 110,60 & 1,64 \\
C. quinquefasciatus + L. durhamii & 41 & 41,00 & 0,48 \\
C. quinquefasciatus + C. bigoti & 15 & 20,90 & 0,55 \\
C. quinquefasciatus + L. durhamii + C. bigoti & 6 & 14,50 & 0,49 \\
C. quinquefasciatus + L. durhamii + Toxorhynchites sp. & 6 & 3,83 & 0,34 \\
L. durhamii & 24 & 95,50 & 1,57 \\
L. durhamii + presa & 16 & 51,40 & 1,27 \\
L. durhamii + C. bigoti + presa & 6 & 39,50 & 1,22 \\
L. durhamii + Toxorhynchites sp. + presa & 6 & 65,33 & 1,50 \\
\hline
\end{tabular}

Aplicando-se o Teste de Análise de Variância, para comparar as quantidades de imaturos coletados de Culex quinquefasciatus e Culex eduardoi, quando coabitavam com os diferentes predadores, obteve-se resultado significativo em nível de $5 \%$. Esta constatação pode indicar a eficiência dos três predadores em diminuir a população de Culex quinquefasciatus e Culex eduardoi.

Toxorhynchites é o gênero mais estudado, visando a sua utilização como agente de controle biológico. Neste experimento não apareceu coabitando somente com as espécies consideradas presas, sempre havia uma das espécies predadoras junto. Todavia, sempre que presente, a densidade populacional da presa ficava significativamente diminuída. TRPIS (1972), em área suburbana na África, descobriu que em pneus com Toxorhynchites brevipalpis Theobaldi, 1901 a população de Aedes aegypti (Linnaeus, 1762) era reduzida para menos de um terço em relação à encontrada nos pneus sem o predador. LouniBOs et al. (1987) observaram diminuição significativa na densidade populacional de Culicidae, Ceratopogonidae, Psychodidae, Thaumaleidae e Chironomyidae. 
Limatus durhamii não pode ser indicada como um agente eficaz de controle biológico, e isto se justifica por ser uma espécie predadora não obrigatória. Como esta espécie, eventualmente, pode exercer um papel predatório, era de se esperar que sua população devesse ser maior, quando estivesse coabitando com as espécies consideradas presas, pois seria evitado o canibalismo, sendo preferidas para alimentação as larvas de outras espécies. Os resultados observados foram contrários, nos criadouros onde havia somente as larvas de Limatus durhamii a média foi de 95,5 e onde coabitava com as presas foi de 51,4 larvas (Tab. I). Este desvio pode estar na dependência das diferentes épocas do ano. As espécies-presas foram mais abundantes na primavera e a predadora no verão. Assim, os encontrados em coexistências foram mais freqüentes na primavera, quando o potencial reprodutivo de Limatus durhamii ainda era baixo.

A maior freqüência de Limatus durhamii ocorreu no verão, quando a água dos criadouros já tinha mais de seis meses, estando, conseqüentemente, rica em matéria orgânica, o que evitou o canibalismo e manteve a densidade populacional alta. Resultados de coexistência destas e de outras espécies estão detalhadas em LOPES (1997).

LOPES et al. (1985b) obtiveram resultados antagônicos aos observados aqui, sendo que a maior densidade de Limatus durhamii foi em presença de Culex bonnei Dyar, 1921 e não quando sozinha, aparentando que nestas condições estaria evitando o canibalismo e exercendo a predação.

MACHADO-ALLISON (1981) argumentou que o canibalismo facultativo seria uma simples resposta à falta de presa. Outra resposta diferenciada do esperado é um número alto de Limatus durhamii em presença de Toxorhynchites sp., o que poder sugerir uma maior agressividade da primeira, quando comparada com Culex quinquefasciatus e Culex eduardoi, dificultando a ação do predador principal.

Culex quinquefasciatus em presença de Limatus durhamii apresentou média maior que em presença de Culex bigoti, fato inverso foi observado com Culex eduardoi. Isto pode mostrar preferência de Limatus durhamii por Culex eduardoi em comparação a Culex quinquefasciatus e de Culex bigoti por Culex quinquefasciatus. Também a média de Culex quinquefasciatus foi mais baixa na presença de Toxorhynchites quando comparada com a média de Culex eduardoi na presença deste predador.

Transformando-se os dados da tabela $\mathrm{I}$ em $\log (\mathrm{x}+1)$ devido à presença de muitos valores zero, aplicou-se o Teste de Análise de Variância para detectar diferenças estatisticamente significativas. O teste foi significativo a nível de 5\% $(\mathrm{P}<0,05)$, tanto para as variações populacionais de Culex quinquefasciatus em presença dos predadores como para Culex eduardoi.

O Teste de Tukey evidenciou a nível de $5 \%(\mathrm{P}<0,05)$, que Culex quinquefasciatus sozinho diferencia-se de todas as outras coexistências. Por outro lado, as combinações apresentadas formam um grupo único, não indicando diferenças significativas em relação ao tamanho da população da presa, em relação às espécies de predador. Para Culex eduardoi, o Teste de Tukey mostrou que sua população foi significativamente maior quando encontrada isolada no criadouro. Culex bigoti apresenta como o menos eficiente e Limatus durhamii como o mais eficiente na predação daquela espécie. 
Limatus durhamii, na presença das duas outras espécies predadoras, passa a ser presa. Observou-se apenas seis vezes a coexistência com cada uma das predadoras, em cujas associações também se faziam presentes as duas espécies-presas. A população de Limatus durhamii decaiu mais significativamente em presença de Culex bigoti. Embora esta predadora tenha demonstrado maior preferência por Limatus durhamii do que Toxorhynchites sp., ambas as predadoras acusaram preferência por espécies originalmente consideradas presas.

Culex bigoti e Toxorhynchites sp. manifestaram preferência por Culex quinquefasciatus e enquanto Limatus durhamii, por Culex eduardoi. KURIHARA \& ICHIMERI (1975), mencionaram a ação predadora de Culex vorax Edwards, 1921 que, como Culex bigoti, pertence ao subgênero Lutzia Theobaldi, 1903.

\section{REFERÊNCIAS BIBLIOGRÁFICAS.}

ANNIS, B. \& S. RUSMIARTO. 1988. Trial of a quantified mass rearing method for Toxorhyncites splendens. Jour. Amer. Mosq. Control Assoc. 4 (3): 368-369.

BAILEY, O. L.; D. ADABBE; D. FocKS \& D.P. DAME. 1983. Field release with adults and pupae of Toxorhynchites rutilus rutilus (Diptera: Culicidae). Jour. Med. Entomol. 20: 308-312.

CASTNER, J.L. \& D.L. BAILEY. 1984. Effects of Toxorhynchites amboinensis on laboratory reared Aedes aegypti populations (Diptera: Culicidae). Jour. Med. Entomol. 21 (2): 133-136.

ForATtini, O.P. 1965. Entomologia médica. São Paulo, Edusp, Vol. 2, 506p.

Focks, D.A.; S.R. SACKETT \& O.L. BAILEY. 1982. Fields experiments on the control of Aedes aegypti and Culex quinqefasciatus by Toxorhynchites rutilus rutilus (Diptera: Culicidae). Jour. Med. Entomol. 19 (3): 336-339.

HubBaRD, S.F.; S.L.C. O'MALLEY \& R. Russo. 1988. The functional response of Toxorhynchites rutilus rutilus to changes in the density of its prey Aedes aegypti. Med. Vet. Entomol. 2: 279-283.

HutCHINGS, R.S.G. 1995. Conteúdo dos criadouros larvais e comportamento de adultos de Toxorhynchites (Lynchiella) haemorrhoidalis haemorrhoidalis (Fabricius) (Diptera: Culicidae) numa floresta de terra-firme da amazônia central. Revta bras. Zool. 12 (2): 313-319.

Kurihara, T. \& K. ICHIMORI. 1975. Preliminary studies on the bionomics of the immature population of Culex pipiens pallens in na artificial habitat. Jap. Jour. Sanit. Zool. 26 (2-3): 167-169.

LOPES, J. 1997. Ecologia de mosquitos (Diptera: Culicidae) em criadouros naturais e artificiais de área rural no Norte do Paraná, Brasil. VII. Coexistência das espécies. Iheringia, Sér. Zool., Porto Alegre, 83: 91-97.

LOPES, J.; J.R. ARIAS \& J.D. HARLWOOD. 1985a. Estudo ecológico de Culicidae (Diptera) silvestres criando em pequenos recipientes de água em mata e em capoeira no Município de Manaus-Am. Ciêcia e Cultura 37 (8): 1299-1311.

Lopes, J.; J.D. Charlwood \& J.R. ArIas. 1985b. Sobreposição de espécies de Culicidae (Diptera) criando em pequenos recipientes de água em floresta e capoeira no município de Manaus-Am. Rev. Brasil. Biol. 4: 609-614.

Lopes, J.; M.A.N. Da Silva; V.D.R.B. DE Oliveira \& M.C.P. Braga. 1995. Ecologia de mosquitos (Diptera: Culicidae) em criadouros naturais e artificiais 
de área rural do Norte do Paraná, Brasil. III. Viabilização de recipientes como criadouro. Semina, Londrina, 16 (2): 244-253.

Lounibos, L.P.; J.H. Frank; C.E. Machado-Allison; P. Ocanto \& J.C.

NAVARRO. 1987. Survival, development and predtory effects of mosquito larvae in Venezuelan phytatelmata. Jour. Trop. Ecol. 3 (3): 221-247.

MACHADO-ALLISON C.E. 1981. Ecologia de los mosquitos (Culicidae). II. Larvas y pupas. Acta Biol. Venez. 11 (1): 51-129.

O'FLYNN, S.M. \& G.B. CRAIG JR. 1982. Effects of Toxorhynchites brevipalpus on Aedes aegypti (Diptera: Culicidae) in continuous breeding laboratory populations. Jour. Med. Entomol. 19 (4): 380-387.

RUsso, R.J. 1983. The functional response of Toxorhynchites rutilus rutilus

(Diptera: Culicidae), a predator on container- breeding mosquitoes. Jour. Med. Entomol. 20 (6): 585-590.

1986. Comparison of predatory behavior in five spicies of Toxorhynchites

(Diptera: Culicidae). Ann. Entomol. Soc. Amer. 79 (4): 715-722.

TOOHEY, M.K.; M.S. GoETTEL; M. TAKagi; R.C. RAM; G. PRAKash \& J.S. Pillai.

1985. Fields studies on the introduction off the mosquitoes predator Toxorhynchites amboinensis (Diptera: Culicidae) into Fiji. Jour. Med. Entomol. 22 (1): 102-110.

TRPIS, M. 1972. Development and predatory behavior of Toxorhynchites brevipalpis (Diptera: Culicidae) in relation to temperature. Environ. Entomol. 1 (5): 537-546.

Recebido em 14.VII.1998; aceito em 18.VIII.1999. 